\title{
ESTILO DE VIDA NOCTURNO: ¿EPIDEMIA DE LA FALTA DE SUEÑO?
}

\section{NIGHTLIFE STYLE: AN EPIDEMIC OF LACK OF DREAM?}

\section{Osvaldo MELGAREJO ${ }^{1}$.}

${ }^{1}$ Médico residente de Psiquiatría, Facultad de Ciencias Médicas, Universidad Nacional de Asunción, San Lorenzo - Paraguay.

Cómo citar este artículo: Melgarejo O. Estilo de vida nocturno: ¿epidemia de la falta de sueño? Medicina Clínica y Social. 2017;1(3):224-226.

\section{Estimado Editor,}

A medida que el mundo fue adquiriendo nuevas tecnologías y desarrollando nuevos aparatos electrónicos accesibles a la mayoría de las personas, éstas se fueron adaptando a ellos e incluyéndolos en sus rutinas nocturnas como una forma de entretenimiento, lo que terminó por generar un retraso en el inicio del sueño "voluntario". El tipo de luz que emiten los dispositivos electrónicos como teléfonos móviles, tabletas, televisores y computadores portátiles es del rango de la luz azul (1), el cual tiene un impacto sobre el estado de alerta y la fatiga, así como una reducción en la secreción de melatonina $(2,3)$; lo anterior desencadena un retraso en la aparición del sueño, con consecuencias al día siguiente, conocidas por las personas con insomnio, tales como: disfunción del rendimiento cognitivo, irritabilidad, alteración del apetito, el peso y las funciones metabólicas (4-7).

La falta de sueño o el sueño insuficiente es un problema considerado de salud pública en Estados Unidos de América, donde lo relacionan con accidentes automovilísticos, desastres industriales y errores médicos. Uno de los factores causales de esta alteración en el sueño es el acceso a las tecnologías durante las 24 horas $(8,9)$. Además de lo citado anteriormente, el sueño insuficiente (por distintas causas, como los trabajos nocturnos) ha sido estudiado y relacionado de manera positiva con el riesgo aumentado de cáncer de mama en mujeres (10, 11).

Los requerimientos de sueño varían según la edad de las personas, siendo mayor el requerimiento cuanto menor es la edad y mayor el riesgo de muerte temprana cuando se incumplen las horas de sueño necesarias (tanto de menos como de más) (12).

Este "jet lag social" (1) producido por la inclusión de las nuevas tecnologías a las rutinas nocturnas es visto por algunos autores como cazar ninjas en la oscuridad (13), donde la causa del sueño insuficiente está aparentemente oculta en la habitación, pero a la vez se encuentra al alcance de nuestras manos. Si bien las medidas no farmacológicas benefician al $70-80 \%$ de las personas con dificultades crónicas para dormir (14), muchas veces se hace necesario limitar el tiempo que se pasa con aparatos que emiten el tipo de luz descripto precedentemente, al menos 30 minutos antes de ir a dormir $(1,15)$, con el fin de disminuir la latencia en el inicio del sueño, el tiempo total del sueño, y la calidad del sueño y a largo plazo, mejorando finalmente la calidad de vida. Y es en ese sentido, que se podrá atender a la preocupación mundial 
por la salud pública sobre el sueño, que ha aumentado la demanda de estrategias sobre promoción del sueño, que sean eficaces y accesibles a la población toda (16).

\section{CONFLICTOS DE INTERÉS Y FUENTE DE FINANCIACIÓN}

El autor declara no poseer conflictos de interés. Fuente de financiación: ninguna.

\section{REFERENCIAS BIBLIOGRÁFICAS}

1. Touitou Y, Reinberg A, Touitou D. Association between light at night, melatonin secretion, sleep deprivation, and the internal clock: Health impacts and mechanisms of circadian disruption. Life Sci. 2017;173:94-106. https://doi.org/10.1016/j.Ifs.2017.02.008

2. Sinclair KL, Ponsford JL, Taffe J, Lockley SW, Rajaratnam SM. Randomized controlled trial of light therapy for fatigue following traumatic brain injury. Neurorehabil Neural Repair. 2014;28(4):303-313. http://doi.org/10.1177/1545968313508472

3. Duffy JF. Czeisler CA. Effect of Light on Human Circadian Physiology. Sleep Med Clin. 2009;4(2):165-177. https://doi.org/10.1016/j.jsmc.2009.01.004

4. Lamond N, Jay SM, Dorrian J, Ferguson SA, Jones C, Dawson D. The dynamics of neurobehavioural recovery following sleep loss. J Sleep Res. 2007;16(1):33-41. https://doi.org/10.1111/j.1365-2869.2007.00574.x

5. Carskadon, MA. Acebo, C. Seifer, R. Extended nights, sleep loss, and recovery sleep in adolescents. Arch Ital Biol. 2001;139(3):301-312. URL.

6. Schmid S, Hallschmid M, Schultes B. The metabolic burden of sleep loss. The Lancet Diabetes \& Endocrinology 2015;3(1):52-62. https://doi.org/10.1016/S22138587(14)70012-9

7. Dube N, Khan K, Loehr S, Chu Y, Veugelers P. The use of entertainment and communication technologies before sleep could affect sleep and weight status: a populationbased study among children. Int J Behav Nutr Phys Act. 2017;14(1):97. https://doi.org/10.1186/s12966-017-0547-2

8. Krahe A [Internet]. Academy of Clinical Sleep Disorders Disciplines; c2016 [cited 14 Dec 2017]. CDC Declares Insufficient Sleep a "Public Healthcare Problem" [approx. 2 screens]. Available from: http://acsdd.org/2016/12/07/rand-corp-insufficient-sleep/

9. Sleep Foundation [Internet]. Sleepfoundation.org; c2011 [cited 14 Dec 2017]. Annual Sleep in America Poll Exploring Connections with Communications Technology Use and Sleep [approx. 5 screens]. Available from: https://sleepfoundation.org/media-center/press-release/annual-sleep-america-poll-exploring-connections-communicationstechnology-use-

10. Schernhammer ES, Laden F, Speizer FE, Willett WC, Hunter DJ, Kawachi I, et al. Rotating night shifts and risk of breast cancer in women participating in the Nurses Health Study. J Natl Cancer Inst. 2011;93(20):1563-1568. URL.

11. Schernhammer ES, Kroenke $\mathrm{CH}$, Laden F, Hankinson SE. Night work and risk of breast cancer. Epidemiology. 2006;17(1):108-111. URL.

12. Miró E, Cano-Lozano C, Buela-Casal G. Sueño y calidad de vida. Rev. colomb. psicol. 2005;14:11-27. URL.

13. Marshall N. The sleep loss epidemic: hunting ninjas in the dark. Journal of Sleep Research. 2015;24(1):1-2. https://doi.org/10.1111/jsr.12277 
14. Morin CM, Hauri PJ, Espie CA, Spielman AJ, Buysse DJ, Bootzin RR. Nonpharmacologic treatment of chronic insomnia: an American Academy of Sleep Medicine review. Sleep 1999;22(8):1134-1156. URL.

15. Foley L, Maddison R, Jiang Y, Marsh S, Olds T, Ridley K. Presleep Activities and Time of Sleep Onset in Children. Pediatrics. 2013;131(2):276-282. URL.

16. Irish LA, Kline CE, Gunn HE, Buysse DJ, Hall MH. The role of sleep hygiene in promoting public health: A review of empirical evidence. Sleep Med Rev. 2015;22:23-36. https://doi.org/10.1016/j.smrv.2014.10.001 